Rev Biomed 2004; 15:73-79.

\title{
Caracterización de los sitios de multiplicación de Aedes aegypti (Diptera: Culicidae) en el caserio "La Carpio", San José, Costa Rica durante la estación seca del año 2003.
}

Artículo Original

Olger Calderón-Arguedas, Adriana Troyo, Mayra E. Solano.

Centro de Investigación en Enfermedades Tropicales (CIET), Departamento de Parasitología, Facultad de Microbiología, Universidad de Costa Rica, San José, Costa Rica.

\section{RESUMEN.}

Introducción.- El primer brote de dengue en San José, la capital de Costa Rica, fue documentado hasta el año 2002 en la comunidad "La Carpio", donde las condiciones climáticas y sociales son adecuadas para la transmisión viral. La caracterización de los sitios de multiplicación para Aedes aegypti en esta comunidad es esencial para desarrollar estrategias de control y prevención de la transmisión del dengue.

Material y métodos.- Se realizó la inspección de 369 viviendas en los 4 sectores de "La Carpio", en las cuales se identificaron los potenciales criaderos peridomiciliares e intradomiciliares para Ae. aegypti. El material larval colectado fue fijado en alcohol al $70 \%$, aclarado en lactofenol y montado en medio Hoyer, para su observación microscópica.

Resultados.- 242 (65.58\%) de las viviendas estudiadas presentaron potenciales criaderos para $A e$. aegypti. La mayoría de éstos (estañones y baldes) tuvieron una localización peridomiciliar $(\mathrm{p}<0.05)$. Sin embargo, sólo en 19 de las viviendas (5.14\%) fue posible observar depósitos con larvas de Ae. aegypti.
Los contenedores intradomiciliares positivos fueron floreros y macetas, en tanto que los peridomiciliares positivos, en su mayoría, fueron estañones.

Discusión.- Los contenedores grandes, utilizados para el almacenamiento de agua, fueron los más frecuentemente infestados con Ae. aegypti. En el Sector 4 del caserío, donde el suministro de agua en tubería no es tan problemático, tuvieron lugar los índices entomológicos más altos. Esto podría relacionarse con el lento recambio y consumo de agua almacenada, hábito común en la población. La abatización de estos contenedores así como las campañas de información y educación son necesarias para prevenir la multiplicación del vector.

(Rev Biomed 2004; 15:73-79)

Palabras clave: Ae. aegypti, dengue, Culicidae, criaderos de mosquito, Costa Rica.

\section{SUMMARY.}

Aedes aegypti (Diptera: Culicidae) breeding sites

Solicitud de sobretiros: Olger Calderón-Arguedas, Departamento de Parasitología, Facultad de Microbiología, Universidad de Costa Rica, San José, Costa Rica. C Correo electrónico: olgerc@cariari.ucr.ac.cr Recibido el 20/Octubre/2003. Aceptado para publicación el 24/Mayo/2004.

Este artículo está disponible en http://www.uady.mx/sitios/biomedic/revbiomed/pdf/rb041521.pdf

Vol.15/No.2/Abril-Junio, 2004 
O Calderón-Arguedas, A Troyo, ME Solano.

in the neighborhood "La Carpio", San José, Costa Rica during the dry season of 2003.

Introduction: The first dengue outbreak in San José, the capital city of Costa Rica, was documented in 2002 in the community of "La Carpio", where suitable climatic and social conditions are adequate for the viral transmission. The characterization of breeding sites for Aedes aegypti in this community is essential for the development of strategies for vector control and prevention of dengue transmission.

Material and methods: 369 houses were visited in the 4 sectors of "La Carpio", and the presence of outdoor and indoor water filled containers was documented. Mosquito larvae were collected, fixed in $70 \%$ alcohol, mounted in Hoyer's Medium, and observed by light microscope.

Results: 242 (65.58\%) of the houses showed potential breeding sites for Ae. aegypti. Most of the containers (drums and buckets) were located outdoors ( $\mathrm{p}<0.05)$. However, only $19(5.14 \%)$ of the houses had containers positive for Ae. aegypti. The indoor containers more often infested by the vector were flower vases and flowerpots, while the main outdoor positive containers were water drums.

Discussion: Large containers used for water storage were the most frequently infested by Ae aegypti. Sector 4, where the pipe water supply is not a serious problem, showed the highest entomological indices for Ae. aegypti. This could be due to low consumption and exchange of stored water by the population. The utilization of abate in this kind of containers, as well as information and education campaigns are necessary for vector control in this community.

\section{(Rev Biomed 2004; 15:73-79)}

Key words: Ae. aegypti, dengue, Culicidae, mosquito breeding sites, Costa Rica.

\section{INTRODUCCIÓN.}

Durante la primera parte de los años 90, gran cantidad de países ubicados en los trópicos y subtrópicos, a lo largo del mundo, experimentaron la reaparición del dengue en forma de epidemias a gran escala, por lo que esta enfermedad viral se ha convertido en un importante problema de salud pública (1). Su distribución geográfica incluye más de 100 países, donde más de 2.5 billones de personas están en riesgo de infección (2). El principal vector del virus, Aedes aegypti, es un mosquito eminentemente sinantrópico, el cual utiliza contenedores de agua artificiales como los principales sitios de oviposición y multiplicación en los ambientes urbanos (1).

Los procesos de urbanización han condicionado, tanto la proliferación de depósitos de agua de origen humano, como la concentración de individuos susceptibles al virus, por lo que estos aspectos se han relacionado con la frecuencia y magnitud de las epidemias de dengue (3).

En Costa Rica, Ae. aegypti fue erradicado en los años cincuenta, y desde 1971 se han dado reinfestaciones en ciudades como Puntarenas, Liberia y Limón (4). Para principios de la década de los noventa, el mosquito se encontraba distribuido a lo largo de todo el territorio nacional. Llama la atención que, a pesar de la importancia sanitaria que representa la infestación por Ae. aegypti, la información publicada en Costa Rica, en este aspecto, es muy escasa (5).

La epidemia de dengue, iniciada en 1993, ha afectado a gran cantidad de comunidades, principalmente en la Costa Pacífica del país. El casquete urbano de la capital no se relacionó con la transmisión de la virosis, hasta el período comprendido entre Agosto y Noviembre de 2002, el cual coincide con la mayor precipitación pluvial para la estación lluviosa, cuyo promedio llega a alcanzar los $240 \mathrm{~mm}$ en el área capitalina. En este período se documentó el primer brote de dengue en el caserío denominado "La Carpio", cercano al centro urbano de San José. "La Carpio" es un poblado que se ha desarrollado secundariamente a un proceso de invasión de terrenos, con una planificación urbanística precaria. El caserío es habitado por una población en su mayoría nicaraguiense, que muestra una gran movilidad, ya que constituye parte de la fuerza laboral de la región. Además, dentro de sus características ambientales, se debe destacar su temperatura, cuyo promedio anual de $22.6^{\circ} \mathrm{C}$, es dos grados mayor que la

\section{Revista Biomédica}


Caracterización de criaderos de Ae. aegypti en "La Carpio", San José, Costa Rica.

temperatura del Centro Urbano de San José, lo que convierte al lugar en un sitio susceptible para la transmisión de la virosis (6).

El conocimiento de las condiciones reproductivas de Ae. aegypti que prevalecen en esta comunidad es fundamental para el desarrollo de estrategias de control apropiadas. El propósito de este estudio es la identificación y tipificación de los principales contenedores que pueden funcionar como sitios de multiplicación para el mosquito Ae. aegypti en la comunidad de "La Carpio", durante la estación seca, con el fin de identificar cuáles son los factores naturales o derivados de la ecología humana, que podrían condicionar la ocurrencia de nuevos brotes de la enfermedad.

\section{MATERIAL Y MÉTODOS.}

Se realizó una encuesta larval por Ae. aegypti en viviendas pertenecientes al poblado conocido como "La Carpio". El estudio se llevó a cabo en los meses de Marzo y Abril de 2003 que forman parte de la estación seca.

"La Carpio" tiene una ubicación a $7 \mathrm{Km}$ al Oeste del centro urbano de San José (latitud $09^{\circ} 58^{\prime} \mathrm{N}$, longitud $84^{\circ} 08^{\prime} \mathrm{O}$ ). En la estación seca (DiciembreAbril), los promedios de temperatura y precipitación pluvial son de $22.5^{\circ} \mathrm{C}$ y $25.58 \mathrm{~mm}$, respectivamente, en tanto que durante la estación lluviosa (MayoNoviembre) estos valores corresponden a $22.7^{\circ} \mathrm{C}$ y $220 \mathrm{~mm}$.

"La Carpio" cuenta con 2705 viviendas y una población aproximada de 30000 habitantes (cuadro
1). Aunque existen casas cuya construcción se podría tipificar como buena, la mayor parte está constituida por viviendas parcialmente terminadas o de construcción informal. El suministro de agua a la población tiene lugar por un sistema de acueducto, que permite que las viviendas obtengan el líquido directamente de la tubería. La distribución urbana del poblado se ha dividido en cuatro sectores, que han sido definidos por el personal de salud del caserío y tiene como fin la organización e implementación de campañas y políticas de salud.

El muestreo practicado en cada uno de los sectores, fue de tipo sistemático (7) y el tamaño muestral fue definido de acuerdo a los lineamientos establecidos por la Organización Panamericana de la Salud (OPS), utilizando como criterio el índice aédico de viviendas y fijando un intervalo de confianza del 95\% (8) (cuadro 1).

En cada una de las viviendas estudiadas, se contabilizaron y examinaron todos los recipientes adecuados para retener agua de forma no transitoria, tanto en el intra como en el peridomicilio. Cada contenedor fue examinado mediante observación directa y una muestra de las formas larvales de mosquitos presentes fue transferida, mediante la ayuda de cernidores y pinzas entomológicas, a contenedores de cristal de 64 × $7 \mathrm{~mm}$ con alcohol al $70 \%$, para su conservación y transporte al laboratorio. Este material fue aclarado en lactofenol claro y montado entre porta y cubreobjetos en medio Hoyer para su posterior observación al microscopio de luz. La identificación taxonómica de las larvas se llevó a cabo mediante el

\section{Cuadro 1}

Viviendas del Caserío la Carpio que se estudiaron por larvas de Ae. aegypti durante la estación seca de 2003.

\begin{tabular}{ccccc}
\hline $\begin{array}{c}\text { Sector } \\
\text { evaluado }\end{array}$ & $\begin{array}{c}\mathbf{N}^{\circ} \text { de } \\
\text { viviendas }\end{array}$ & $\begin{array}{c}\mathbf{N}^{\circ} \text { de viviendas } \\
\text { evaluadas } \\
\text { del sector }\end{array}$ & $\begin{array}{c}\mathbf{N}^{\circ} \text { de viviendas } \\
\text { con potenciales } \\
\text { criaderos para } \\
\text { Ae. aegypti }\end{array}$ & $\begin{array}{c}\mathbf{N}^{\circ} \text { de viviendas } \\
\text { con infestación } \\
\text { positiva por } \\
\text { Ae. aegypti }\end{array}$ \\
\hline 1 & 698 & 113 & 73 & 5 \\
2 & 651 & 59 & 37 & 3 \\
3 & 684 & 98 & 63 & 3 \\
4 & 672 & 99 & 69 & 19 \\
\hline
\end{tabular}




\section{O Calderón-Arguedas, A Troyo, ME Solano.}

uso de claves dicotómicas especializadas (9). La presencia de larvas de Ae. aegypti permitió designar al contenedor correspondiente como positivo por este díptero. Los índices entomológicos pertinentes: índice de contenedores (IC), índice de viviendas (IV) e índice de Breteau (IB) se calcularon de acuerdo a lineamientos preestablecidos (8). Estos índices fueron calculados con base en las observaciones hechas a todos los contenedores identificados (intra y peridomiciliares) en las viviendas en estudio.

Los porcentajes de depósitos observados, en el intra y en el peridomicilio se compararon mediante pruebas de Z para comparación de proporciones, utilizando un coeficiente de confiabilidad del $95 \%$ (10); además la composición cualitativa de los contenedores observados en cada sector, así como su frecuencia,

fueron evaluadas mediante pruebas de Chi cuadrada de independencia, utilizando un coeficiente de confiabilidad del 95\% (10).

\section{RESULTADOS.}

La presencia de potenciales criaderos para $A e$. aegypti fue observada en 242 (65.58\%) de las 369 viviendas visitadas (cuadro 1), en las cuales se pudieron identificar 573 depósitos con agua acumulada de forma no transitoria, 147 en el intradomicilio y 426 en el peridomicilio (cuadro 2). El porcentaje de contenedores presentes en el peridomicilio fue significativamente mayor al que se observó intradomiciliarmente $(\mathrm{p}<0.05)$ (fig. 1$)$.

La distribución del tipo y cantidad de depósitos en los sectores que componen el caserío, tanto en el Cuadro 2

Contenedores adecuados para la multiplicación de Ae. aegypti que se observaron en el Caserío "La Carpio" durante la estación seca de 2003.

\begin{tabular}{|c|c|c|c|c|c|}
\hline \multirow[t]{2}{*}{ Contenedor } & \multicolumn{4}{|c|}{$\begin{array}{l}\text { Intradomicilio } \\
\text { Sector }\end{array}$} & \multirow[t]{2}{*}{ Total } \\
\hline & 1 & 2 & 3 & 4 & \\
\hline Estañón & $36(43.90)^{*, \dagger}$ & $1(5.55)$ & $13(68.42)$ & $9(32.14)$ & $59(40.14)^{\ddagger}$ \\
\hline Balde & $30(36.60)$ & $6(33.33)$ & $4(21.05)$ & $13(46.43)$ & $53(36.05)$ \\
\hline Maceta & $6(7.31)$ & $0(0.00)$ & $0(0.00)$ & $1(3.57)$ & $7(4.76)$ \\
\hline Florero & $3(3.66)$ & $7(38.89)$ & $0(0.00)$ & $3(10.71)$ & $13(8.84)$ \\
\hline Lata & $2(2.44)$ & $1(5.55)$ & $1(5.26)$ & $0(0.00)$ & $4(2.72)$ \\
\hline Otros & $5(6.10)$ & $3(16.67)$ & $1(5.26)$ & $2(7.14)$ & $11(7.48)$ \\
\hline Total & $82(100.00)$ & $18(100.00)$ & $19(100.00)$ & $28(100.00)$ & $147(100.00)$ \\
\hline \multirow[t]{2}{*}{ Contenedor } & \multicolumn{4}{|c|}{$\begin{array}{l}\text { Peridomicilio } \\
\text { Sector }\end{array}$} & Total \\
\hline & 1 & 2 & 3 & 4 & \\
\hline Estañón & $46(41.82) *$, & $6(10.53)$ & $26(20.31)$ & $44(33.59)$ & $122(28.64)$ \\
\hline Balde & $25(22.73)$ & $26(45.61)$ & $36(28.13)$ & $32(24.43)$ & $119(27.93)$ \\
\hline Maceta & $1(0.91)$ & $0(0.00)$ & $5(3.91)$ & $2(1.53)$ & $7(1.64)$ \\
\hline Botella & $7(6.36)$ & $0(0.00)$ & $7(5.47)$ & $4(3.05)$ & $18(4.23)$ \\
\hline Llanta & $1(0.91)$ & $7(12.28)$ & $3(2.34)$ & $4(3.05)$ & $15(3.52)$ \\
\hline Pila & $1(0.91)$ & $2(3.51)$ & $1(0.78)$ & $2(1.53)$ & $6(1.41)$ \\
\hline Bebedero de animales & $6(5.45)$ & $2(3.51)$ & $0(0.00)$ & $0(0.00)$ & $8(1.88)$ \\
\hline Coco & $1(0.91)$ & $0(0.00)$ & $3(2.34)$ & $11(8.40)$ & $15(3.52)$ \\
\hline Lata & $2(1.82)$ & $4(7.02)$ & $16(12.50)$ & 14 (10.69) & $36(8.45)$ \\
\hline Otros & $20(18.18)$ & $10(17.54)$ & $31(24.22)$ & $18(13.74)$ & $80(18.78)$ \\
\hline Total & $110(100.00)$ & $57(100.00)$ & $128(100.00)$ & $131(100.00)$ & $426(100.00)$ \\
\hline
\end{tabular}

* La prueba de Chi cuadrada de independencia determinó la ocurrencia de una distribución no homogénea en las frecuencias observadas $(\mathrm{p}<0.05)$.

$\uparrow$ Porcentaje por sector

¥ Porcentaje del total

Revista Biomédica 


\section{Caracterización de criaderos de Ae. aegypti en "La Carpio", San José, Costa Rica.}

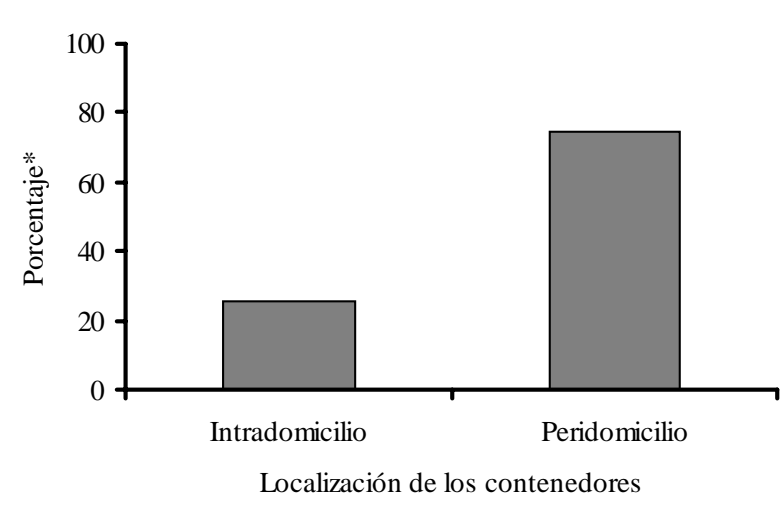

Figura 1.- Localización de los contenedores de agua potencialmente permisivos para la multiplicación de Ae aegypti observados en la comunidad de "La Carpio".

* Los porcentajes de contenedores del intra y peridomicilio mostraron diferencias estadísticamente significativas $(\mathrm{p}<0.05)$

intra como en el peridomicilio, no fue homogénea $(\mathrm{p}<0.05)$. El Sector 1 fue el que presentó la mayor cantidad de contenedores intradomiciliares, mientras que el Sector 4 mostró la mayor cantidad de depósitos peridomiciliares. De forma global, los contenedores más frecuentes tanto en el intra como en el peridomicilio fueron los estañones y baldes (cuadro 2).

El porcentaje de viviendas con contenedores

positivos por Ae. aegypti fue sólo del 5.14\% (19 viviendas). Los floreros y las macetas fueron los únicos contenedores intradomiciliares positivos por $\mathrm{Ae}$. aegypti que se observaron, mientras que los estañones constituyeron los depósitos más importantes en lo relativo a presencia de larvas de Ae. aegypti en el peridomicilio. Cabe destacar que en el Sector 3 no fue posible observar contenedores intradomiciliares positivos por larvas del vector (cuadro 3 ).

El Sector 4 fue el que presentó la mayor infestación por formas larvales de Ae. aegypti, por lo que sus índices aédicos respectivos fueron los que mostraron los valores más elevados. (cuadro 4).

\section{DISCUSIÓN.}

A pesar de que Ae. aegypti puede utilizar como criaderos algunos reservorios de agua naturales como huecos de árboles, cáscaras de frutas o bromelias, los sitios preferenciales están constituidos por contenedores artificiales $(1,11)$. El caserío "La Carpio" presenta un ambiente que facilita la presencia de lugares potencialmente adecuados para la colonización de Ae. aegypti, ya que exhibe una alta concentración de población, una escasa planificación urbana y limitaciones en la recolecta de basura y el Cuadro 3

Contenedores positivos por larvas de Ae. aegypti observados en el Caserío "La Carpio" durante la estación seca de 2003.

\begin{tabular}{|c|c|c|c|c|c|}
\hline \multirow[t]{2}{*}{ Contenedor } & \multicolumn{4}{|c|}{$\begin{array}{l}\text { Intradomicilio } \\
\text { Sector }\end{array}$} & \multirow[t]{2}{*}{ Total } \\
\hline & 1 & 2 & 3 & 4 & \\
\hline$\overline{\text { Maceta }}$ & $1(100.00)^{*, \dagger}$ & $0(0.00)$ & $0(0.00)$ & $1(100.00)$ & $2(50.00)^{\ddagger}$ \\
\hline Florero & $0(0.00)$ & $2(100.00)$ & $0(0.00)$ & $0(0.00)$ & $2(50.00)$ \\
\hline Total & $1(100.00)$ & $2(100.00)$ & $0(0.00)$ & $1(100.00)$ & $4(100.00)$ \\
\hline \multirow[t]{2}{*}{ Contenedor } & \multicolumn{2}{|c|}{ Sector } & & & Total \\
\hline & 1 & 2 & 3 & 4 & \\
\hline Estañón & $1(25.00)^{*, \dagger}$ & $2(100.00)$ & $0(0.00)$ & $6(75.00)$ & $9(52.94)^{\ddagger}$ \\
\hline Balde & $2(50.00)$ & $0(0.00)$ & $2(66.66)$ & $0(0.00)$ & $4(23.53)$ \\
\hline Maceta & $1(25.00)$ & $0(0.00)$ & $0(0.00)$ & $2(25.00)$ & $3(17.65)$ \\
\hline Lata & $0(0.00)$ & $0(0.00)$ & $1(33.33)$ & $0(0.00)$ & $1(5.88)$ \\
\hline Total & $4(100.00)$ & $2(100.00)$ & $3(100.00)$ & $8(100.00)$ & $17(100.00)$ \\
\hline
\end{tabular}

Vol.15/No.2/Abril-Junio, 2004 
O Calderón-Arguedas, A Troyo, ME Solano.

Cuadro 4

Índices entomológicos para Ae. aegypti observados en los sectores que conforman el caserío "La Carpio".

\begin{tabular}{lcccc}
\hline \multirow{2}{*}{ Índice } & \multicolumn{4}{c}{ Sector } \\
\cline { 2 - 5 } & $\mathbf{1}$ & $\mathbf{2}$ & $\mathbf{3}$ & $\mathbf{4}$ \\
\hline Indice de Contenedores $^{*}$ & 2.60 & 5.33 & 2.04 & 5.66 \\
Índice de Viviendas $^{\dagger}$ & 4.42 & 5.08 & 3.06 & 8.08 \\
Índice de Breteau $^{*}$ & 4.42 & 6.78 & 3.06 & 9.09 \\
\hline
\end{tabular}

*contenedores positivos por Ae. aegypti/contenedores evaluados x 100

'viviendas con contenedores positivos por Ae. aegyptil viviendas evaluadas $\mathrm{x} 100$

contenedores positivos por Ae. aegypti/viviendas evaluadas x 100

suministro de agua.

En 242 de las 369 casas que se evaluaron en el presente estudio se pudieron identificar 573 depósitos (147 en el intradomilio y 426 en el peridomicilio) que acumulaban agua de forma no transitoria y pueden ser adecuados para la oviposición del vector. Los contenedores que se observaron con mayor frecuencia fueron los estañones y los baldes, que son utilizados por los pobladores para el almacenamiento de agua. En estudios realizados en países como Brasil, se ha visto que durante la estación seca éstos son los contenedores que tienen mayor relevancia en cuanto a permitir la multiplicación del vector y mantener densidades vectoriales adecuadas para que se dé la transmisión del dengue (1). En este sentido, se ha demostrado que la asociación directa entre precipitación/densidad larval e incidencia de dengue, aunque suele ser frecuente, no siempre se da (12). Esta es la razón por la cual este tipo de depósitos adquieren una importancia epidemiológica fundamental durante las estaciones con poca precipitación.

Los índices entomológicos observados en el presente estudio fueron bajos en comparación con los observados en otros estudios que se han llevado a cabo en comunidades urbanas durante la estación seca $(13,14)$. En dos comunidades de la Provincia del Chaco Argentina, se observaron, durante la estación seca, los siguientes índices aédicos: IV entre 67 y $70 \%$, IC entre 18 y $39 \%$ e IB entre 127 y más de 200, que son francamente superiores a los observados en el presente estudio (13). En otra comunidad centroamericana denominada "El Progreso" en Honduras, se pudieron documentar índices de Breteau entre 32 y 25 para la época seca (14), que también excedieron a los evidenciados en el caserío "La Carpio".

Los bajos índices entomológicos observados en el poblado en estudio sugieren que las densidades vectoriales en esta época del año son bajas (cuadro 4). Los únicos depósitos intradomiciliares positivos por el vector fueron floreros y macetas (cuadro 3), mientras que en el peridomicilio los estañones fueron los contenedores más frecuentemente infestados por el vector (cuadro 2). Estos resultados pueden obedecer a la exhaustiva campaña que han implementado las autoridades de salud de la localidad ante una situación post epidémica, en la cual se ha incluido la remoción de la basura no tradicional, el saneamiento ambiental y la educación de la población. Estos aspectos son fundamentales en lo que respecta a la disminución de las densidades larvales en las comunidades, con efectos positivos superiores a los alcanzados con las campañas tradicionales basadas en el rociamiento de insecticidas (14).

El Sector 4, el cual presentó la mayor cantidad de potenciales criaderos en el peridomicilio, mostró los índices entomológicos más elevados, aun siendo el sector con menos problemas en el suministro de agua potable en tubería. Esto puede deberse a que, al igual que en los otros sectores, la práctica de almacenaje de agua es común; sin embargo, al ser el suministro de agua adecuado, el recambio y consumo de la misma se da con menos frecuencia, lo que facilita el establecimiento de formas inmaduras de Ae. aegypti. En este sentido, en un estudio realizado en un poblado rural de Venezuela, se pudo observar que la interrupción del servicio de agua en tubería tenía una correlación positiva con los índices de viviendas y de Breteau. Sin embargo, aproximadamente un 60\% de los pobladores reconoció la costumbre de seguir manteniendo agua almacenada, aun cuando se diera el reestablecimiento del servicio (15).

Para garantizar el control de la transmisión del dengue en comunidades como la estudiada, se requiere

\section{Revista Biomédica}




\section{Caracterización de criaderos de Ae. aegypti en "La Carpio", San José, Costa Rica.}

de una adecuada vigilancia epidemiológica, así como la implementación de campañas que promuevan la eliminación de criaderos, el saneamiento ambiental, la educación a la población y la abatización de depósitos utilizados en el almacenamiento de agua.

\section{AGRADECIMIENTOS.}

Los autores agradecen al Dr. Oscar Montero, a la Lic. Fredda Wilhem y al personal asistencial del EBAIS de la comunidad "La Carpio" por la colaboración prestada; a los estudiantes Annia Mejía, José Luis Vargas, Graciela Oguilve, Viviana Araya e Israel Aragón que participaron en el proyecto TC 492 por su labor operativa y a la sección de Trabajo Comunal Universitario de la Vicerrectoría de Acción Social en la Universidad de Costa Rica por su apoyo económico y logístico a este trabajo.

\section{REFERENCIAS.}

1.- Pinheiro VCS, Tadei WP. Frequency, diversity, and productivity study on the Aedes aegypti most preferred containers in the city of Manaus, Amazonas, Brazil. Rev Inst Med Trop S Paulo 2002; 44:245-50.

2.- Lopes de Fonseca BA, Szent-Fonseca SN. Dengue viral infections. Curr Opin Pediatr 2002; 14:67-71.

3.- Service MW. Importance of Ecology in Aedes aegypti control. Southest Asean J Trop Med Publ Health 1992; 23:6819.

4.- Guzmán MG, Huelva G, Sáenz E, Quiróz E, de los Reyes J, Balmaseda A, et al. Reintroducción del dengue 3 en las Américas 1994-1996. Virol 1998; 2:8-19.

5.- Recio-Domingo M, Ruiz-Díaz JI, Figueroa-Córdoba D, Hernández-Chavarría F. Epidemiología del dengue en el Cantón de Esparza, Puntarenas, Costa Rica. 1997-2002. Rev Cos Cien Med 2002; 23: 141-6.

6.- Fundación Arias para la Paz, Centro para las Naciones Unidas para los Asentamientos Humanos CNUAH-Hábitat, Internacional Development Research Center. La población migrante nicaragüense en Costa Rica: realidades y respuestas. San José: Imp Obando; 2000.

7.- Nelson M. The role of sampling in vector control. Am J Trop Med Hyg 1994; 50: 145-50.

8.- Organización Panamericana de la Salud. Dengue y dengue hemorrágico en las Américas, Publicación Científica 548. OPS Washington (DC) OPS; 1997.
9.- Capernter SJ, La Classe WJ. Mosquitoes of North America. Los Angeles: University of California Press; 1955. p. 144-7.

10.- Daniel W. Bioestadística: Base para el análisis de las ciencias de la salud. 3a ed. México: Editorial Limusa; 1988. p. 459-502.

11.- Tabachnick W. The yellow fever mosquito. Amer Entomol 1991; 37:14-24.

12.- Kuno G. Review of the factors modulating dengue transmission. Epidemiol Rev 1995; 17: 321-35.

13.- Stein M, Oria GI. Identificación de criaderos de Ae. aegypti (Díptera: Culicidae) y cálculo de índices de infestación en la provincia del Chaco. En: Salomón, O, editor. Actualizaciones en artropodología sanitaria argentina. Buenos Aires: Fundación Mundo Sano; 2002. p. 161-6.

14.- Leonstsini E, Gil E, Kendall C, Clark GG. Effect of a community-based Aedes aegypti control programme on mosquito larval production sites in El Progreso, Honduras. Trans Roy Soc Trop Med Hyg 1993; 87: 267-71.

15.- Barrera R, Avila J, González-Tellez S. Unreliable supply of potable water and elevated Ae. aegypti larval indices: a causal relationship? J Am Mosq Contr Assoc 1993; 9: 18995. 